\title{
Urgences
}

\section{Jean Simard, Félix, Montréal, Stanké, « Québec 10/10 », no 87} (1947), 1986, 154 p.

\section{Guy Rancourt}

Numéro 14, août 1986

Corps et jouissances

URI : https://id.erudit.org/iderudit/025271ar

DOI : https://doi.org/10.7202/025271ar

Aller au sommaire du numéro

\section{Éditeur(s)}

Urgences

\section{ISSN}

0226-9554 (imprimé)

1927-3924 (numérique)

Découvrir la revue

Citer ce compte rendu

Rancourt, G. (1986). Compte rendu de [Jean Simard, Félix, Montréal, Stanké, "Québec 10/10 », no 87 (1947), 1986, 154 p.] Urgences, (14), 81-83.

https://doi.org/10.7202/025271ar d'utilisation que vous pouvez consulter en ligne.

https://apropos.erudit.org/fr/usagers/politique-dutilisation/ 
Mais la récupération du voisinage a ses limites: comme Judith Krantz qu'il cite, Rohmer incline à penser que les gens réels sont ennuyeux, exception faite de leurs moments de passion. Qu'il faudrait seuls utiliser.

Le style sera personnel; quelle qu'en soit la venue, se rappeler qu'il charrie deux fonctions: la communication et l'entertainment. On produira la première version - le brouillon - d'instinct, sans autocensure... mais dans le cadre du plan. Rohmer prône l'écriture de plusieurs versions de l'oeuvre. Rewriting. Pour ce faire, un conseil: Show, don't tell! Montrer plutôt que dire. Rewriting.

Le futur écrivain adulé empruntera aux jurons et au langage sexuel avec attention. En assaisonner son récit, n'en pas faire un plat... principal. L'intrigue commandera cette inclusion d'épices. Se rappeler qu'on vise le plus large public possible... Par ailleurs, point de discipline, point de best-seller. La gestion optimale du temps s'impose! Time is money. Les presses toument... Se faire un horaire d'écriture, y tenir! Nul succès sans travail. S'isoler, se concentrer, créer son environnement: Rohmer a un appartement en Floride quand celui de Toronto ne convient pas..

L'éditeur interviendra avant la version finale. Il est votre objectivité. L'éditeur de best-sellers conseille, aide à la recherche, oriente le travail, corrige le manuscrit - ne devrait-on pas dire machinescript? Nous sommes loin des imprimeurs qui se prennent pour des éditeurs. La version finale sera le fait d'un travail d'équipe: l'écrivain, les recherchistes, l'éditeur, les lecteurs-conseil... et, bien sûr, l'agent. Futurs écrivains célèbres, il vous faut un agent littéraire, un imprésario, lequel négociera vos contrats, organisera votre promotion et vos entrevues avec les média nationaux, trouvera des débouchés à vos oeuvres: théâtre. cinéma, télévision, conférences... Le best-seller établira le pont entre la littérature et le show busjness! Et alors les lucarnes lumineuses s'ouvriront pour vous. Et les gens vous verront partout. Ils vous achèteront le lendemain. D'autant plus volontiers que le concept de votre livre stimulera leur consommation des modes.

Au Québec, tous les préalables identifiés par Rohmer sont-ils réunis qui favoriseraient notre part de best-sellers? Que je sache, aucune version québécoise ni française - d'un livre similaire à How to Write a Best Sellern'existe! Existeraitelle qu'elle s'avérerait peut-être une deuxième - hors Le Matou point de salut! - occasion d'éditer un gros vendeur.

Gilbert Dupuis, juin 86

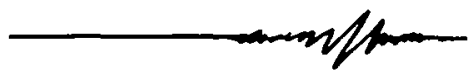

Jean Simard, Félix, Montréal, Stankē, "Québec 10/ 10", no 87 (1947), 1986, $154 \mathrm{p}$.

FÉLIX? Ah oui! Fêlix Leclerc ou Félix le chat, tout dépendant si notre locuteur est âgé ou jeune. Ah oui! c'est la remise annuelle des trophées Félix, nous diront plutôt les mordus de musique, d'arts et spectacles. Or l'amoureux de notre littérature (qu'il soit ou non fin connaisseur ou spécialiste chevronné) saura qu'il s'agit aussi du premier roman publié par Jean Simard en 1947. Jean Simard??? Connais pas! Peut-être, me direz-vous? Ah sacrée mémoire! Et pourtant, cet écrivain, natif de Québec (1916), nous a 
légué entre 1947 et 1983 onze publications ( 5 romans, 3 essais, 2 recueils de récits, contes et nouvelles et une pièce de théâtre) et a traduit une dizaine d'oeuvres littéraires d'auteurs canadiens-anglais.

Revenons donc à Félix. Ce petit roman connut dès sa publication un franc succès. On lui attribua le Prix Kornmain de l'Académie française en 1947. On le réédita en 1966 aux Éditions Estérel. Enfin, après un long silence de vingt ans, Roch Carrier eut l'heureuse idée de le rééditer en édition de poche, c'est-ădire dans la collection "Québec 10/10" chez Stanké. Les rééditions de Félix, comme des deux oeuvres, depuis longtemps introuvables de Clément Marchand, Courriers des villages (1942) et Les soirs rouges (1947), m'apparaissent fort intéressantes et démontrent le flair et la finesse de Roch Carrier vis-àvis de notre littérature. Rééditer les jeunes écrivains, certes, mais ne point oublier nos aînés aussi! Relever ce défi, c'est assurer, en somme, la continuité et la permanence de notre littérature...

Fellx, sous-titré LIvre d'enfant pour adultes et illustré par l'auteur, est le récit drolatique d'un jeune Québécois des années 40. Jean Simard nous présente son héros en quarante-quatre courts chapitres de 2 ou 3 pages. (Il y a du peintre là-dessous - 44 petites retouches sont nécessaires pour fabriquer un croquis ou une ébauche de portrait!) Toutefois, un "Avis au lecteur" nous en dit long sur l'étroitesse des mentalités de l'époque. On nous met tout de suite en garde: "les péchés de Fêlix ne sauraient être imputés à l'auteur qui est, lui, un monsieur sérieux, sévère et respectueux des lois" (p. 7). Le lecteur, vite rassuré de l'hiatus entre l'auteur et 'le héros, pourra à son aise tout connaître de Félix. De ses lointaines origines (pâle descendant de La Vérendrye par métissage), de son grand-père notaire malheureux en affaires, des fiançailles et épousailles de ses parents, de sa conception maladroite et antivoluptueuse, de son bâptême et de sa première communion, de son précepteur privé Gérald Goulu et de leur bonne Clara, de ses études et de ses maîtres, de la mort de son père, de ses convictions religieuses, de ses marivaudages, etc. Bref, Jean Simard laissera son héros sur le deuil de ses vingt ans au sortir "de l'imbécile et ineffable adolescence" ( $p$. 134).

Féllx, à proprement parler, n'est pas un véritable roman. Cela ressemble davantage à un récit-essai. Amplement truffé d'épigraphes (les 44 chapitres débutent par une citation d'auteur), de proverbes et maximes, d'observations et réflexions philosophiques, Félix (tout comme les autres romans de Jean Simard, sauf La séparation) annonce déjà l'oeuvire magistrale de l'essayiste qui signera tour à tour Répertoire (1961), Nouveau Répertoire (1965) et Une façon de parler (1973).

Je relis sa dernière oeuvre, Le singe et le perroquet (1983), et ces 45 petits récits me ramènent inévitablement à Félix espiègle, cabotin, cynique et philosophe, sauf que Félix semble avoir gagné en maturité, vieilli et qu'il raconte cette fois-ci la suite de son "imbécile et ineffable" vie adulte. Et ne croyez surtout pas que l'auteur en est dupe car, après la relecture de Féllkx, Jean Simard constate "le fait que ce premier livre, mon "livre de jeunesse" (signalons que 
l'auteur avait tout de même 31 ans!), ressemble déjà terriblement à des Mémoires!" (p. 141).

Reste à savoir comment le jeune lou encore le moins jeune) Québécois des années 1980 s'identifiera ou accueillera ce héros velléitaire qui prône tantôt le "dilettantisme libertin", tantôt le "paradoxe", ou tout simplement l'ironie et le sarcasme pour affronter la vie. Vie qu'il définit comme "une prison garnie de barreaux: d'un côté les gardiens; de lautre les détenus. Mais qui me dira lesquels, en vérité, sont les captits des autres?" (p. 135). C'est sur cette vision et interrogation concernant la vie que Félix se termine.

La lecture/relecture de Félix présente donc un réel intérêt pour le lecteur québécois en tant que véritable miroir de ses contradictions, ambivalences, dualités; bref, de sa si difficile quête d'identité.

\section{Guy Rancourt Cégep de Rimouski}

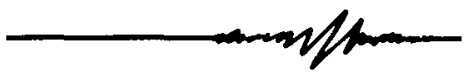

\section{Michel Serres, Les cing sens, Philosophie des corps mêlés I, Paris, Grasset, 1985, 381 p.}

Qu'il écrive à propos de tel peignoir peint par Bonnard, qu'il s'adonne à l'observation fascinée d'une peau ou d'un voile sur une nudité, d'un tissu ou d'une brume, qu'il commente la Dame à la licome ou qu'il pose avec ironie. devant la chaussure de vair de Cendrillon, des questions à la fois sérieuses et ingénues - "Que dire, d'abord, d'une pantoufle? Donnez, je vous prie, votre estime, au passage, à un livre de philosophie qui pose enfin les graves questions - que dire, dis-je, d'une pantoufle?" (p. 64) - c'est à une véritable philosophie de la sensation, à un érotisme de la texture, de la mixture et du grain auquel nous convie Michel Serres dans Les cinq sens. D'ailleurs le soustitre, Philosophie des corps mêlés, désigne de lui-même cette attention qui sera portée à tout ce qui se fusionne, s'immisce, s'emmêle, s'imbrique; et l'essai de Serres assiste à ce travail de la métamorphose et de l'osmose qui constitue l'univers en une matière intensément tangible, en une sorte de "pâte" malléable par tous les sens et offerte à tous les possibles du désir et de la jouissance. Ainsi, le goût du vin participe de l'échange et de la transfusion: "Si vous le goûtez, il vous donnera votre goût en vous donnant son goût, il ouvre en vous une nouvelle bouche" (p. 168). De même, tout regard reçoit vision du spectacle qu'il contemple: "[...] une belle vue offre en plus la vue à qui la voit" (p. 168).

Portée par une culture qui relève de l'encyclopédie, - mais une culture "naturelle", dont l'étendue n'a d'égal que la sensualité - la philosophie, ici, s'intéresse avec une même curiosité et un même tact aux arts, aux sciences, à la littérature; passant de Socrate à Leibnitz, de Hermès à Oedipe, elle réfléchit aussi bien aux mythes qu'aux modes, aux saveurs, d'un fruit, d'un vin - "une bouteille de vieil Yquem, année 1947" (p. 165) - qu'aux nuances d'un bleu ou à celles d'un paon. Alors la pensée ne se démêle plus du sensible et le goût, tant esthétique que gastronomique, s'avoue dans le savoir de la jouissance. Certes, il y a du lyrisme et de l'euphorie dans le point de vue du philosophe. Aussi. dans le ravissement des sens qu'il 